\title{
FACTORS AND ROOTS OF THE VAN DER POL POLYNOMIALS
}

\author{
F. T. HOW ARD
}

ABSTRACT. The van der Pol polynomials $V_{n}(a)$ are defined by means of

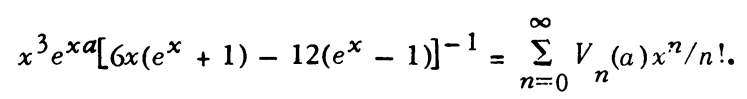

In this paper new properties of these polynomials are derived. It is shown that neither $V_{2 n}(a)$ nor $V_{2 n+1}(a) /(a-1 / 2)$ has rational roots, and that if $n=2 \cdot 3^{m}, m \geq 0$, or $n=3^{m}+3^{t}, m>t>0$, or $n=m(p-3), p$ a prime number, $3 m<p$, then $V_{n}(a)$ and $V_{n+1}(a) /(a-1 / 2)$ are both irreducible over the rational field. It is also shown that if $n=2^{k}$, then $V_{n}(a)$ is irreducible over the rational field. Finally, possible factors of the van der Pol polynomials are discussed.

1. Introduction. The van der Pol numbers $V_{0}, V_{1}, V_{2}, \cdots$ can be defined by means of the generating function

$$
\frac{x^{3}}{6 x\left(e^{x}+1\right)-12\left(e^{x}-1\right)}=\sum_{n=0}^{\infty} V_{n} \frac{x^{n}}{n !} .
$$

This definition is apparently due to B. van der Pol [12], who used these numbers in a problem concerning the smoothing and unsmoothing of functions of three variables. The present writer pointed out [7] that the van der Pol numbers are closely related to the Rayleigh function $\sigma_{2 n}(\nu)$, a function which is defined in terms of the zeros of the Bessel function and which has been the subject of a number of investigations by Kishore [8], [9], Carlitz [2], [4], and others. (The early history of the Rayleigh function can be found in [14, p. 502].) In fact, for $n>1, \sigma_{2 n}(3 / 2)=(-1)^{n-1} 3 \cdot 2^{2 n-1} V_{2 n} /(2 n) !$. In [7] the writer defined the van der Pol polynomial $V_{n}(a)$ by means of

$$
\frac{x^{3} e^{x a}}{6 x\left(e^{x}+1\right)-12\left(e^{x}-1\right)}=\sum_{n=0}^{\infty} V_{n}(a) \frac{x^{n}}{n !} \text {. }
$$

It follows that $V_{n}(0)=V_{n}$, and

$$
V_{n}(a)=\sum_{r=0}^{n}\left(\begin{array}{l}
n \\
r
\end{array}\right) V_{r} a^{n-r},
$$

Received by the editors September 27, 1974.

AMS (MOS) subject classifications (1970). Primary 10A40; Secondary 12D05, 12D10.

Key words and phrases. van der Pol numbers and polynomials, Rayleigh function, Bernoulli and Euler polynomials, irreducible over the rational field, Eisenstein's irreducibility criterion, Eisenstein polynomial. 
In [6] it was proved that for $n>0, V_{2 n}(a)$ has exactly two real roots between 0 and 1 , and $V_{2 n+1}(a)$ has exactly three real roots, including $a=1 / 2$, between 0 and 1 . It was also shown that $V_{n}(a)$ has no nonreal roots on the lines $x=0, x=1 / 2$, and $x=1$ in the complex plane. Thus it appears that the van der Pol polynomials have properties similar to those of the well-known Bernoulli and Euler polynomials. See [1], [3], [10, Chapter 2], and [11], for example. The first eight van der Pol polynomials are listed in $\$ 5$.

In the present paper we continue our investigation of the roots and rational factors of the van der Pol polynomials. In particular we prove the following:

(a) If $n$ is even then $V_{n}(a)$ has no rational roots.

(b) If $n$ is odd then $V_{n}(a) /(a-1 / 2)$ has no rational roots.

(c) Neither $V_{2 n}(a)$ nor $V_{2 n+1}(a) /(a-1 / 2)$ has a factor of the form $c_{0}+$ $c_{1} a+\cdots+c_{k} a^{k}$ where $c_{0}, \ldots, c_{k}$ are integers, $k>0, c_{k} \equiv 0(\bmod 5)$.

(d) If $V_{2 n}(a)$ or $V_{2 n+1}(a) /(a-1 / 2)$ has a factor of the form $c_{0}+c_{1} a+$ $\cdots+c_{k} a^{k}, k>0$, where $c_{0}, \ldots, c_{k}$ are integers, then if $c_{0} \not \equiv 0(\bmod 5)$, we must have $c_{i} \equiv 0(\bmod 5)$ for all $i>0$.

(e) If $n=2 \cdot 3^{m}, m \geq 0$, or $n=3^{m}+3^{t}, m>t>0$, or $n=m(p-3)$ where $p$ is a prime and $3 m<p$, then $V_{n}(a)$ and $V_{n+1}(a) /(a-1 / 2)$ are irreducible over the rational field.

(f) If $n=2^{k}, k>0$, then $V_{n}(a)$ is irreducible over the rational field.

2. Preliminaries. The van der Pol numbers are rational and the exact powers of 2,3, and 5 dividing the denominator of $V_{n}$, when $V_{n}$ is reduced to its lowest terms, are known [6], [7]. Because this information will be useful when we examine the roots and factors of $V_{n}(a)$, we incorporate it into the following lemma. Since $2 V_{2 n+1}=-(2 n+1) V_{2 n}$ for $n>1$ [7], we shall state the lemma for $V_{2 n}$. Similar congruences obviously hold for $V_{2 n+1}$.

Lemma 2.1. (a) If $n>1$ then $2 V_{2 n} \equiv 1(\bmod 4)$.

(b) If $n>1$ then for $b=0,1,2,3 V_{2 n} \equiv b(\bmod 3)$ if $2 n \equiv b+1(\bmod 3)$.

(c) If $n>0$ then $5^{n} V_{2 n} /(2 n) ! \equiv 3^{n}(\bmod 5)$.

(d) If $p$ is a prime, $p>3$, then for $n>0, p^{n} V_{n(p-3)} /[n(p-3)] ! \equiv(-12)^{n}$ $(\bmod p)$.

When proving irreducibility theorems, we shall use the following version of Eisenstein's criterion.

Lemma 2.2. If $f(x)=c_{0}+c_{1} x+\cdots+c_{n} x^{n}$ is a polynomial with integer coefficients and if there is a prime $p$ such that $c_{0} \equiv 0(\bmod p), c_{i} \equiv 0(\bmod$ $p)$ for all $i>0, c_{n} \not \equiv 0\left(\bmod p^{2}\right)$, then $f(x)$ is irreducible over the rational field.

Suppose $f(x)$ is a polynomial with rational coefficients. If there is an 
integer $h$ such that $h f(x)$ has integer coefficients and satisfies the hypotheses of Lemma 2.2, then we shall call $f(x)$ an Eisenstein polynomial. Clearly an Eisenstein polynomial is irreducible over the rational field.

Lemma 2.3. If $f(x)=c_{0}+c_{1} x+\cdots+c_{n} x^{n}$ is a polynomial with integer coefficients and if there is a prime $p$ such that $c_{0} \equiv 0(\bmod p), c_{i} \equiv 0(\bmod$ p) for all $i>0$, then if $f(u / b)=0$ where $u$ and $b$ are integers, $(u, b)=1$, we must have $b \equiv 0(\bmod p)$.

Proof. It is easy to see that if $f(x)$ satisfies the above hypotheses, then $u \not 0(\bmod p)$. Now suppose $b \not 0(\bmod p)$. We can write

$$
f(x)=(b x-u)\left(r_{0}+r_{1} x+\cdots+r_{n-1} x^{n-1}\right)
$$

where each $r_{i}$ is a rational number reduced to its lowest terms. Now, using induction on $i$, we can show that each $r_{i}$ is integral $(\bmod p)$; that is, $p$ does not divide the denominator of $r_{i}$. This is true for $i=0$ since $c_{0}=b r_{0}$ is an integer. Assume true for $i=k-1$; then $c_{k}=b r_{k-1}-u r_{k}$, and since $c_{k}$ is an integer, $r_{k}$ must be integral $(\bmod p)$. Now, looking at $f(x)(\bmod p)$, we see that $b x-u$ divides $c_{0}(\bmod p)$, which is impossible (see [13, pp. 74-77]).

Throughout this paper we use the notation $p^{k} \| c$ to mean $p^{k}$ is the highest power of $p$ dividing $c$.

Lemma 2.4. Let $f(x)=c_{0}+c_{1} x+\cdots+c_{n} x^{n}$ be a polynomial of degree $n>3$ with nonzero integer coefficients. Suppose $f(x)$ has all the following properties:

(a) There is a prime $p$ such that $c_{0} \equiv 0(\bmod p), c_{i} \equiv 0(\bmod p)$ for all $i>0$.

(b) If $p^{k_{i}} \| c_{i}, i>0$, then $k_{n-3} \geq k_{n-2}, k_{i} \geq k_{i+1}-1, k_{i} \geq k_{i+2}-1$.

(c) $f(a)=0$ implies $f(1-a)=0$.

Then we can conclude that $f(x)$ has no rational roots.

Proof. We know from Lemma 2.3 that if $f(u / b)=0$, then $b \equiv 0(\bmod p)$. By hypothesis (c) we can write

$$
f(x)=(b x-u)(b x-b+u)\left(r_{0}+r_{1}+\cdots+r_{n-2} x^{n-2}\right)
$$

where each $r_{i}$ is a rational number reduced to its lowest terms. Since

$$
c_{i}=u(b-u) r_{i}-b^{2} r_{i-1}+b^{2} r_{i-2},
$$

we can prove by induction on $i$ that each $r_{i}$ is integral $(\bmod p)$. Now we show that if $p^{k} \| c_{i}$, then $p^{k} \|$ numerator of $r_{i}$. This is true for $i=0$ since $c_{0}=u(b-u) r_{0}$. Suppose it is true for all $j<i$. Then we have $c_{i}=$ $\dot{u}(b-u) r_{i}-b^{2} r_{i-1}+b^{2} r_{i-2}$, and by part (b) of our hypotheses we must have $p^{k} \|$ numerator of $r_{i}$. Now since 


$$
c_{n-2}=b^{2} r_{n-4}-b^{2} r_{n-3}+u(b-u)_{n-2} \text { and } c_{n-1}=b^{2} r_{n-3}-b^{2} r_{n-2} \text {, }
$$

we have a contradiction of hypothesis (b). Therefore $f(u / b) \neq 0$.

We shall make use of the well-known facts in the following lemma [5, pp. 263, 271].

Lemma 2.5. If $p$ is a prime and

$$
\begin{array}{ll}
m=a_{0}+a_{1} p+\cdots+a_{n} p^{n} & \left(0 \leq a_{i}<p\right), \\
r=b_{0}+b_{1} p+\cdots+b_{n} p^{n} & \left(0 \leq b_{i}<p\right),
\end{array}
$$

then

$$
\left(\begin{array}{l}
m \\
r
\end{array}\right) \equiv\left(\begin{array}{l}
a_{0} \\
b_{0}
\end{array}\right)\left(\begin{array}{l}
a_{1} \\
b_{1}
\end{array}\right) \cdots\left(\begin{array}{l}
a_{n} \\
b_{n}
\end{array}\right) \quad(\bmod p)
$$

(b) If $p^{k} \| m$ ! then $k=\left(m-a_{0}-a_{1}-\cdots-a_{n}\right) /(p-1)$.

3. Rational roots and factors of the van der Pol polynomials. By using Lemma 2.4, we shall prove that neither $V_{2 n}(a)$ nor $V_{2 n+1}(a) /(a-1 / 2)$ has rational roots.

Theorem 3.1. For $n \geq 0$,

$$
\begin{aligned}
5^{n} V_{2 n}(a) /(2 n) ! & \equiv 3^{n} \quad(\bmod 5) \\
5^{n} V_{2 n+1}(a) /(2 n+1) ! & \equiv 3^{n-1}+3^{n} \cdot a \quad(\bmod 5) .
\end{aligned}
$$

Proof. By (1.3) we have

$$
\begin{aligned}
\frac{5^{n} V_{2 n}(a)}{(2 n) !}= & \sum_{r=0}^{n} \frac{5^{r} V_{2 r}}{(2 r) !} \cdot \frac{5^{n-r}}{(2 n-2 r) !} a^{2 n-2 r} \\
& +\sum_{r=0}^{n-1} \frac{5^{r} V_{2 r+1}}{(2 r+1) !} \cdot \frac{5^{n-r}}{(2 n-2 r-1) !} a^{2 n-2 r-1} .
\end{aligned}
$$

Now by Lemma 2.5 (b) we see that if $5^{k} \|(2 n-2 r)$ ! or $5^{k} \|(2 n-2 r-1)$ !, then $k<n-r$ except for the case $r=n$. Thus by Lemma 2.1(c) we have

$$
5^{n} V_{2 n}^{\prime}(a) /(2 n) ! \equiv 5^{n} V_{2 n} /(2 n) ! \equiv 3^{n} \quad(\bmod 5) .
$$

The proof is similar for $V_{2 n+1}(a)$.

Corollary. For $n \geq 0$,

$$
5^{n} V_{2 n+1}(a) /(2 n+1) !(a-1 / 2) \equiv 3^{n} \quad(\bmod 5) .
$$

Theorem 3.2. If $n$ is even then $V_{n}(a)$ has no rational roots. If $n$ is odd then $V_{n}(a) /(a-1 / 2)$ has no rational roots. 
Proof. When $n$ is even, we see by Theorem 3.1 that all the hypotheses of Lemma 2.4 are met and $V_{n}(a)$ has no rational roots. If $n$ is odd, we see by the corollary to Theorem 3.1 and by Lemma 2.3 that if $u / b$ is a root of $V_{n}(a) /(a-1 / 2)$, then $b \equiv 0(\bmod 5)$. We can now proceed as we did in the proof of Lemma 2.4 to show that $V_{n}(a) /(a-1 / 2)$ has no rational roots.

Theorem 3.3. If either $V_{2 n}(a)$ or $V_{2 n+1}(a) /(a-1 / 2)$ has a factor of the form $i(a)=c_{0}+c_{1} a+\cdots+c_{k} a^{k}$ where $c_{0}, \ldots, c_{k}$ are integers, $k>0, c_{0} \not \equiv$ $0(\bmod 5)$, then $c_{i} \equiv 0(\bmod 5)$ for all $i>0$.

Proof. Suppose $V_{2 n}(a)$ does have such a factor $f(a)$ where $c_{m} \not \equiv 0(\bmod$ 5) for some $m>0$. Then we can write

$$
5^{n} V_{2 n}(a) /(2 n) !=f(a) \cdot g(a)
$$

where

$$
g(a)=r_{0}+r_{1} a+\cdots+r_{2 n-k} a^{2 n-k},
$$

each $r_{i}$ a rational number reduced to lowest terms. Since each coefficient of $5^{n} V_{2 n}(a) /(2 n) !$ is integral ( $\left.\bmod 5\right)$, and since the coefficient of $a^{i}$ in (3.2) is $r_{i} c_{0}+r_{i-1} c_{1}+\cdots+r_{0} c_{i}$, we can use induction on $i$ to prove that $r_{i}$ is integral (mod 5). Thus by Theorem 3.1 we see that $f(a)$ divides 1 ( $\bmod 5)$, a contradiction. The proof for $V_{2 n+1}(a) /(a-1 / 2)$ is similar.

Theorem 3.4. Neither $V_{2 n}(a)$ nor $V_{2 n+1}(a) /(a-1 / 2)$ has a factor of the form $f(a)=c_{0}+c_{1} a+\cdots+c_{k} a^{k}$ where $c_{0}, \cdots, c_{k}$ are integers, $k>0, c_{k} \not \equiv$ $0(\bmod 5)$.

Proof. Suppose $V_{2 n}(a)$ does have such a factor $f(a)$. Then again we can write (3.2) and (3.3). Since the coefficient of $a^{k+i}$ in (3.2) is $c_{k} r_{i}+c_{k-1}{ }^{r} i+1$ $+\cdots$ we can use induction on $i$ to prove that $r_{2 n-k-i}$ is integral (mod 5). Thus by Theorem 3.1 we see that $f(a)$ divides $1(\bmod 5)$, a contradiction. The proof for $V_{2 n+1}(a) /(a-1 / 2)$ is similar.

4. Some cases of irreducibility. Carlitz [3] used Eisenstein's irreducibility criterion to obtain results concerning the irreducibility of the Bernoulli and Euler polynomials. With the aid of Lemma 2.1, we shall use the same method on the van der Pol polynomials. It is perhaps useful to recall that $V_{n}(a)$ is monic.

Theorem 4.1. Let $n=m(p-3)$ where $p$ is a prime and $3 m<p$. Then $V_{n}(a)$ and $V_{n+1}(a) /(a-1 / 2)$ are irreducible over the rational field.

Proof. We have

$$
p V_{n}(a)=p \sum_{r=0}^{n}\left(\begin{array}{c}
(m-1) p+p-3 m \\
r
\end{array}\right) V_{r} a^{n-r} .
$$


In [7] it is proved that if $r$ is in any of the half open intervals $[0, p-3)$, $[p, 2(p-3)), \ldots,[(m-1) p, m(p-3))$, then $V_{r}$ is integral $(\bmod p)$. Also

$$
p^{2} V_{r} \equiv 0 \quad(\bmod p), \quad 0 \leq r \leq m(p-3) .
$$

Thus by Lemma 2.5 (a) and Lemma 2.1 (d) we have

$$
p V_{n}(a) \equiv p V_{n} \not \equiv 0 \quad(\bmod p) .
$$

Thus $V_{n}(a)$ is an Eisenstein polynomial, and in a similar way we can also show that $V_{n+1}(a) /(a-1 / 2)$ is an Eisenstein polynomial. In that case we first prove that

$$
p^{m} V_{n+1}(a) /(n+1) ! \equiv a(-12)^{m}+6(-12)^{m-1} \quad(\bmod p)
$$

so

$$
p^{m} V_{n+1}(a) /[(n+1) !(a-1 / 2)] \equiv(-12)^{m} \quad(\bmod p),
$$

and the theorem follows.

Theorem 4.2. If $n=3^{m}+3^{t}, m>t>0$, or if $n=2 \cdot 3^{m}, m \geq 0$, then $V_{n}(a)$ and $V_{n+1}(a) /(a-1 / 2)$ are irreducible over the rational field.

Proof. Let $n=3^{m}+3^{t}$ or $n=2 \cdot 3^{m}$. In either case, by Lemma 2.5 (a) and Lemma 2.1 (b) we have

$$
3 V_{n}(a)=3 \sum_{r=0}^{n}\left(\begin{array}{l}
n \\
r
\end{array}\right) V_{r} a^{n-r} \equiv 3 V_{n} \equiv 2 \quad(\bmod 3) .
$$

Thus $V_{n}(a)$ is an Eisenstein polynomial. Also

$$
3 V_{n+1}(a) \equiv 2 a+2 \quad(\bmod 3), \quad 3 V_{n+1}(a) /(a-1 / 2) \equiv 2 \quad(\bmod 3)
$$

and so $3 V_{n+1}(a) /(a-1 / 2)$ is an Eisenstein polynomial.

Theorem 4.4. If $n=2^{k}, k \geq 0$, then $V_{n}(a)$ is irreducible over the ration. al field.

Proof. By Lemma 2.5 (a) and Lemma 2.1 (a) we have

$$
2 V_{n}(a)=2 \sum_{r=0}^{n}\left(\begin{array}{c}
2^{k} \\
r
\end{array}\right) V_{r} a^{n-r} \equiv 2 V_{n} \equiv 1 \quad(\bmod 2),
$$

since

$$
\left(\begin{array}{c}
2^{k} \\
r
\end{array}\right) \equiv 0 \quad(\bmod 4) \quad \text { if } r \text { is odd. }
$$

We can now see that $V_{n}(a)$ is an Eisenstein polynomial and the theorem follows.

If we examine $V_{n}(a)$ for $0 \leq n \leq 100$, the theorems of this section tell 
us that if $n$ is even, $n \neq 22,46,62,66,72,74,82,92,98$, then $V_{n}(a)$ is irreducible over the rational field. Also, if $n$ is odd, $n \neq 23,47,63,67,73$, $75,83,93,99$, then $V_{n}(a) /(a-1 / 2)$ is irreducible over the rational field.

5. The first 8 van der Pol polynomials.

$$
\begin{aligned}
& V_{0}(a)=1 \\
& V_{1}(a)=a-\frac{1}{2} \\
& V_{2}(a)=a^{2}-a+\frac{1}{5} \\
& V_{3}(a)=a^{3}-\frac{3}{2} a^{2}+\frac{3}{5} a-\frac{1}{20} \\
& V_{4}(a)=a^{4}-2 a^{3}+\frac{6}{5} a^{2}-\frac{1}{5} a-\frac{1}{350}, \\
& V_{5}(a)=a^{5}-\frac{5}{2} a^{4}+2 a^{3}-\frac{1}{2} a^{2}-\frac{1}{70} a+\frac{1}{140}, \\
& V_{6}(a)=a^{6}-3 a^{5}+3 a^{4}-a^{3}-\frac{3}{70} a^{2}+\frac{3}{70} a+\frac{1}{1050}, \\
& V_{7}(a)=a^{7}-\frac{7}{2} a^{6}+\frac{21}{5} a^{5}-\frac{7}{4} a^{4}-\frac{1}{10} a^{3}+\frac{3}{20} a^{2}+\frac{1}{150} a-\frac{1}{300} .
\end{aligned}
$$

\section{REFERENCES}

1. J. Brillhart, On the Euler and Bernoulli polynomials, J. Reine Angew. Math. 234 (1969), 45-64. MR 39 \#4117.

2. L. Carlitz, A sequence of integers related to the Bessel functions, Proc. Amer. Math. Soc. 14 (1963), 1-9. MR 29 \#3425.

3. - Note on irreducibility of the Bernoulli and Euler polynomials, Duke Math. J. 19 (1952), 475-481. MR 14, 163.

4. - Recurrences for the Rayleigh functions, Duke Math. J. 34 (1967), 581-590. MR $35 \# 5670$.

5. L. E. Dickson, History of the theory of numbers. Vol. 1, Publication no. 256, Carnegie Institution of Washington, Washington, D. C., 1919.

6. F. T. Howard, Properties of the van der Pol numbers and polynomials, J. Reine Angew. Math. 260 (1973), 35-46. MR 47 \#6603.

7. - The van der Pol numbers and a related sequence of rational numbers, Math. Nachr. 42 (1969), 89-102. MR 41 \#3385.

8. N. Kishore, The Rayleigh function, Proc. Amer. Math. Soc. 14 (1963), 527533. MR $27 \# 1633$.

9. - The Rayleigh polynomial, Proc. Amer. Math. Soc. 15 (1964), 911917. MR $29 \# 6079$. 1924.

10. N. E. Nörlund, Vorlesungen über Differenzrechnung, Springer-Verlag, Berlin,

11. R. Spira, The nonvanishing of the Bernoulli polynomials in the critical strip, Proc. Amer. Math. Soc. 17 (1966), 1466-1467. MR $34 \# 2967$. 
12. B. van der Pol. Smoothing and "unsmoothing", Probability and Relate Topics in Physical Sciences, Interscience, New York, 1957, pp. 223-235.

13. B. van der Waerden, Modern algebra, Springer, Berlin, 1937; English transl., Ungar, New York, 1949. MR 10, 587.

14. G. N. Watson, A treatise on the theory of Bcssel functions, 2nd ed., Cambridge Univ. Press, Cambridge; Macmillan, New York, 1944. MR 6, 64.

DEPARTMENT OF MATHEMATICS, WAKE FOREST UNIVERSITY, WINSTON-SALEM, NORTH CAROLINA 27109 